tumor (DNT), 70\% had postoperative partial seizures arising in the ipsilateral hemisphere, but $60 \%$ had additional generalized seizures, cognitive, and behavioral disturbance, with multifocal and generalized EEG abnormalities. Nine (20\%) patients had immediate seizure-free periods of at least 1 year, and 7 of these had MTS. On relapse of the 7, 4 had ipsilateral and 3 had contralateral temporal seizures. After postoperative MRI, only one missed structural lesion was uncovered, and reoperations were possible in a minority of cases. The majority of seizures with MTS were extrahippocampal. In the majority of surgical failures, the epileptogenic lesion was either extrahippocampal or extratemporal. Acoustic auras and EEG evidence of neocortical seizure origin in some might have indicated a preoperative poor outcome. Emerging improved MRI may demonstrate subtle cortical abnormalities responsible for operative failures. Patients should be counselled concerning the unpredictable nature of postoperative relapse. (Hennessy MJ, Elwes RDC, Binnie CD, Polkey CE. Failed surgery for epilepsy. A study of persistence and recurrence of seizures following temporal resection. Brain December 2000;123:2445-2466). (Respond: Robert DC Elwes, Department of Clinical Neurophysioiogy, Kings College Hospital, Mapother liouse, de Crespigny Park, London SE5 9RS, UK).

COMMENT. Despite extensive electroclinical and neuroimaging evaluation of temporal lobe epilepsy, approximately $20 \%$ relapse with persistent and recurrent seizures postoperatively, either immediately or after a 12 month seizure-free interval. Until more sophisticated MRI techniques are developed, patients should be aware of the unpredictable outcome.

\title{
DEVELOPMENTAL. DISORDERS
}

\section{PET STUDIES OF LISSENCEPHALY}

The functional activity of lissencephalic cortex was studied using FD Glucose positron emission tomography in 8 patients, mean age 7.5 years, at Children's Hospital of Michigan, Detroit, MI. Two layers of cerebral cortex were differentiated by metabolic activity: an inner layer with 8 to $63 \%$ higher glucose utilization rate than the outer layer. Patients with a higher metabolic ratio between the inner/outer layers had greater delays in communication $(p=.007)$ and socialization $(\mathrm{p}=, 03)$. No difference was found with respect to motor skills. PET studies should provide a more complete analysis of gyral anomalies and clinical outcome in lissencephaly compared to neuroimaging alone. (Pfund Z, Chugani HT, Juhasz C et al. Lissencephaly. Fetal pattern of glucose metabolism on positron emission tomography. Neurology December (1 of 2) 2000;55:1683-1688). (Reprints: Dr Harry T Chugani, Division of Pediatric Neurology/PET Center, Children's Hospital of Michigan, 3901 Beaubien Blvd, Detroit, Ml 48201).

COMMENT. PET studies in lissencephaly show that larger metabolic differences between inner and outer cortical layers are associated with greater delays in communicative skills and socialization. The degree of gyral anomaly in lissencephaly is directly correlated with the degree of developmental delay. The majority of patients with type 1 lissencephaly develop epilepsy before age 6 months, and one-third have infantile spasms.

\section{SURGICAL MANAGEMENT OF CHIARI TYPE 1 ANOMALIES}

A minimally invasive, posterior fossa bony decompression in the management of symptomatic Chiari type 1 anomalies in children was evaluated at 
the Children's Hospital, Turin, Italy. Of 53 patients observed, 27 were asymptomatic and treated conservatively, and 26 had symptoms, associated with brain stem compression in 16 and syringomyelia in 10. Of the group with brain stem compression, $44 \%$ had neck pain, $31 \%$ vertigo, and $31 \%$ headache. In those with syringomyelia, the commonest symptoms were numbness (50\%), sensory loss (40\%), neck pain (40\%), and vertigo (40\%). With an average follow-up after surgery of 22 months (6-60 months), symptoms improved or resolved in all but 3 patients, with no serious complications. A simple extradural surgical approach appears to have comparable results with more aggressive procedures and less complications. (Genitori L, Peretta P, Nurisso C et al. Chiari type 1 anomalies in children and adolescents: minimally invasive management in a series of 53 cases. Child's Nerv Syst Nov 2000;16:707-718). (Dr Genitori, Division of Pediatric Neurosurgery, Children's Hospital (OIRM), P Polonia 94, Turin, Italy).

COMMENT. Surgery for Chiari type 1 is controversial. The American Association of Neurological Surgeons Pediatric Section suggested in 1988 that decompression may be indicated for brain stem or cranial nerve dysfunction associated with the anomaly (Haines SJ, Berger M. Neurosurgery 1991;28:353-357). In symptomatic cases the aim of posterior fossa decompression and cervical laminectomy is to restore a normal CSF circulation at the foramen magnum. The authors find that the simple extradural surgical approach can provide symptomatic relief in most cases, and is superior to more aggressive techniques described in the literature. The following case report of complete and spontaneous resolution of childhood Chiari 1 malformation and associated syringomyelia is noteworthy.

Spontaneous resolution of Chiari I anomaly. A 7-year-old boy with a one month history of severe headaches and vomiting and a family history of migraine was found to have on MRI a Chiari I malformation and cervical syringomyelia. Treated conservatively as migraine with an incidental anomaly, headaches improved following dietary modification and omission of chocolate. At age 13, headaches recurred with greater severity, despite medication. A repeat MRI was normal, showing no evidence of either anomaly. At 16 years, he continues to have headaches every 3 months despite prophylactic Inderal. His neurologic examination is normal. (Sun PP, Harrop J, Sutton LN. Complete spontaneous resolution of childhood Chiari I malformation and associated syringomyelia. Pediatrics Jan 2001;107:182-185). The authors advocate less prophylactic decompression and more frequent conservative management, with serial imaging and neurologic monitoring, in asymptomatic childhood Chiari 1 malformation with associated small or moderate sized syringomyelia. In this case the anomaly was apparently not the cause of the headaches.

\section{VASCULAR DISORDERS}

\section{LUMBAR PUNCTURE WITH ALL AND THROMBOCYTOPENIA}

The potential association of neurologic, infectious, or hemorrhagic complications with lumbar puncture (L.P) during remission induction or consolidation treatment of acute lymphoblastic leukemia (ALL) with thrombocytopenia was determined at St Jude Children's Research Hospital, Memphis, TN. The records of 958 consecutive patients, ages 1 month to 18 years (median, 5.5 years), with newly diagnosed ALL treated between Feb 1984 and July 1998 , were reviewed retrospectively, with special attention to the platelet count at the time of L.P. Of 895 L.Ps performed at diagnosis, serious complications were 